\title{
Subject and language index
}

In this index, genealogical classification details are provided for languages and language subfamilies of South Asia, as follows:

DR: Dravidian

IA: Indo-Aryan (a branch of Indo-European)

LI: $\quad$ language isolate

MD: Munda (a branch of Austroasiatic)

ST: $\quad$ Sino-Tibetan

For example, "Darma (ST)" is the Sino-Tibetan language Darma and "Western Pahari (<IA)" is the Western Pahari subfamily/branch of the Indo-Aryan language family.

Non-South-Asian languages and language (sub)families are not specified in this way, for example "Danish" and "Slavic (language family)". For obvious reasons, Kanashi, Kinnauri, SinoTibetan, and Indo-Aryan are not indexed, although some of the subbranches of ST and IA are, in particular the two main branches of West Himalayish, Eastern and Western West Himalayish (both Kanashi and Kinnauri belong under the latter branch).

See also the "Chapter overview" introducing each chapter.

ablaut, 216, 217

areal linguistics, 49, 237, 240, see also Indosphere, see also language contact, see also Sinosphere

Austroasiatic (language family), 184

Bajjika, see Maithili (IA)

Bantawa (ST), 103

Baram (ST), 185, 186

Bengali (IA), 153

Bhadrawahi (IA), 153, 196

Bhagati (IA), 198

Bhalesi (IA), 16

Bhateali (IA), 153

biodiversity, 7

Bodic ( $<\mathrm{ST}), 5$

Bodish (<ST), 5, 186, 225, 251

Boto Boli, see Raute (ST)

Brahui (DR), 185, 194, 226

Bunan (ST), 5, 47-49, 73, 155-159, 162, 179, $180,184-186,188,224,230,238,248$, 251

Burushaski (LI), 184, 185

Burushaski distribution, 248

Byangsi (ST), 103, 184, 186, 190, 191, 199, 224-228, 238

Cameali (IA), 153
Central Pahari (<IA), 196, 227

Chamba Lahuli, see Tinani (ST)

Chambeali (IA), 196

Chaudangsi (ST), 58, 184-186, 224-228, 238

Chepang (ST), 58

Chhitkuli (ST), 179, 180, 184, 186, 187, 224, 228, 230, 238, 240, 241

Chinali (IA), 185, 196, 227, 228

clusivity, 79, 86, 87, 115, 116, 118, 124

compound

- noun, 58

- numeral, 152

- verb, 123

contact linguistics, see areal linguistics, see language contact

Curahi (IA), 153

Czech, 163

Danish, 148, 163

Dardic (<IA), 159, 198

Darma (ST), 47-49, 103, 119, 152, 184, 186, 190, 199, 224, 225, 238

decimal, see numeral system

Denjongke (ST), 162, 165

deoghar, 160, 161

Dhangar, see Kurukh (DR)

Dolakha Newar, see Newar (ST)

2Open Access. (C) 2022 Anju Saxena and Lars Borin, (c) By-Nc-ND published by De Gruyter. This work is licensed under a Creative Commons Attribution-NonCommercial-NoDerivatives 4.0 International License. https://doi.org/10.1515/9783110703245-010 
Dravidian (language family), 184, 185, 194 , 199, 201-203, 226, 251

Dulong (ST), 103

Dzongkha (ST), 162

Eastern Pahari (<IA), 196

Eastern Panjabi, see Punjabi (IA)

English, 6, 7, 57, 117, 140, 164, 216

evidentiality, 225

exclusive, see clusivity

Finnish, 148, 162

French, 148

Gaddi (IA), 196, 198, 199, 202

Garhwali (IA), 185, 227, 228, 246

German, 163

Germanic (language family), 216

grammaticalization, 101, 115, 216, 229

Gujarati (IA), 153, 202

Gurung (ST), 185, 186

Himachali, see Western Pahari $(<\mid A)$

Himalayish (<ST), 186, 251

Hmong-Mien (language family), 49

honorificity, 78, 79, 115, 126

inclusive, see clusivity

Indosphere, 49, 50

infinitive, 103, 122, 221, 225, 227, see also nominalization, see also participle

Inner Siraji, see Kullu Pahari (IA)

Jad (ST), 186, 189, 193, 225, 248, 251

Jangrami (ST), 241, 248

Japanese, 140

Jaunsari (IA), 16, 73, 185, 196

Kangri (IA), 185, 196

Kath-Kuni, 241, 243, 244, see also Koti Banal

Kathmandu Newar, see Newar (ST)

Khaling (ST), 103

Kinnauri Pahari (IA), 73, 178, 179, 184, 196, 197, 199, 227, 228, 230

Kishtawaarii (IA), 201

Kiunthali (IA), 196

Koci (IA), 179
Kotgarhi (IA), 16, 178, 179, 185, 196, 227, 228

Kotguru (IA), 153

Koti Banal, 241, 243, 244, see also Kath-Kuni Kullu Pahari (IA), 6, 7, 14, 132, 133, 140, 146 , 151, 154, 155, 165, 169, 178, 179, 185, 196-198, 246, 251

Kullui, see Kullu Pahari (IA)

Kulluvi, see Kullu Pahari (IA)

Kumaoni/Kumauni (IA), 185, 191, 196, 227, 228

Kurukh/Kurux (DR), 194, 199, 201, 226, 251

Kusunda (LI), 184, 195, 199, 227, 228, 251

Ladakhi (ST), 185, 186, 248

Lahnda (IA), 196

Lahuli (ST), 47-49, 246

language contact, $6,47,57,134-136,160$, $161,163,165,166,184,188,227,239$, 240, 251, see also areal linguistics

language standardization, 135, 164, 166

Latin, 163

light verb, see support verb, see vector verb

Limbu (ST), 103

Lower Sorbian, see Sorbian

Macro-Tani (<ST), 106

Magar (ST), 186, 193

Mahakiranti (<ST), 186, 193

Mahasu Pahari (<IA), 196

Maithili (IA), 185, 196, 227, 228

Malto (DR), 201

Manchad, see Pattani (ST)

Mandeali (IA), 155, 196

Marathi (IA), 202

MIA, see Middle Indo-Aryan

Middle Indo-Aryan, 174, 179, 200, 202, 216, 239

Moghol, 162

Mon-Khmer (language family), 49

Mongolic (language family), 162

Munda (language family), 184, 194, 203, 204

Mundari (MD), 203, 204

Navajo, 135

Navakat (ST), 73, 76, 179, 180, 184-186, 202, 224, 248

Nepali (IA), 174, 185, 193, 196, 204, 226 
New Indo-Aryan, 174, 200, 202, 216, 230, 239

Newar (ST), 57, 119, 186, 189, 193, 224-226, 228, 230, 251

NIA, see New Indo-Aryan

Nishi (ST), 103

nominalization, 73, 102, 103, 204, 225, see also infinitive, see also participle

Northwestern (<IA), 196, 198, 201

Norwegian, 37, 163

numeral system

- decimal, 148, 149, 151, 152, 156, 157, 159, 162,165

- overcounting, 148, 162

- vigesimal, 148-152, 156, 157, 159, 162, 164, 165

Nyamkad (ST), 185, 186

OIA, see Old Indo-Aryan

Old Indo-Aryan, 174, 179, 200, 202, 216

Old Iranian, 200

Old Swedish, 163, 164

overcounting, see numeral system

Padam-Mishing (ST), 103

participle, 96, 99, 109, 110, see also infinitive, see also nominalization

Pattani (ST), 47-49, 65, 116, 119, 184-186, 188,238

Persian, 57, 162

Poguli (IA), 196, 198, 201

Prakrit (IA), 179

Proto-Indo-European, 164, 200

Proto-Indo-Iranian, 200

Proto-Sino-Tibetan, 26, 44, 47, 49, 156, 159, 216, 239

Proto-West Himalayish (ST), 251

Punjabi (IA), 153, 196

Purik (ST), 185, 186

Qiang (ST), 119

Raji (ST), 184, 186, 191-193, 204, 212, 216, 224-226, 228

Raji-Raute (<ST), 186, 189, 191, 192, 199, 224 , 225, 251

Rambani (IA), 196, 198

Rangkas (ST), 152, 185, 186, 238
Raute (ST), 186, 191-193, 204, 212, 213

Rawang (ST), 103

Rawat (ST), 186, 191, 192, 204, 212, 213

reduplication, 109, 218, 219, 221, 241

Romani (<IA), 201

Rongpo (ST), 103, 184, 186, 189, 190, 199, $224,225,228,238,248,251$

Saadri (IA), 227, 228

Sanskrit (IA), 153, 178, 179, 195, 202

Santali (MD), 194

SAP, see speech act participant

Scottish Standard English, see English

Shumcho (ST), 184, 186-188, 224, 226, 228, 230, 238-241

Shumcho data (ST), 187

Sikkimese, see Denjongke (ST)

Sinosphere, 49, 50

Sirmauri (IA), 196

Slavic (language family), 163

Slovak, 163

Slovene, 163

Sora (MD), 203, 204

Sorbian, 163

speech act participant, 116, 117, 122, 241

Spiti (ST), 248

standard language, see language standardization

Standard Maithili, see Maithili (IA)

Sunnami (ST), 238, 241, 248, 251

suppletion, $116,120,122$

support verb, 57, 102, 122, 227, 230

Swadesh list, 191, 192

Swedish, 147, 163-165

Tai-Kadai (language family), 49

Telugu (DR), 202, 203

Thangmi (ST), 185, 186

Thulung (ST), 103

Thulung Rai (ST), 103

Tibetan (ST), 156, 188, 216, 224, 225, 248

Tibetic (<ST), 179, 241, 246, 248

Tinani (ST), 65, 92, 152, 179, 180, 184-186, $188,224,238$

Tongan, 165

Torwali (IA), 196, 198

transhumance, 161, 248 
Upper Sorbian, see Sorbian

vector verb, 123

Vedic (IA), 184

vigesimal, see numeral system

West Himalayish ( $\angle \mathrm{ST})$

- Eastern, 5, 186, 188, 190, 238, 248, 251

- Western, 5, 186, 228, 238-240, 246, 248 , 249, 251
Western Pahari (<IA), 16, 73, 151, 155, 159, 196, 198, 227

Wintu, 162

Wintuan (language family), 162

Yakkha (ST), 185, 186

Zhangzhung (ST), 5, 157, 158, 238, 248, 251 